https://helda.helsinki.fi

\title{
Anti-neuronal anti-bodies in patients with early psychosis
}

\section{Mantere, $\mathrm{O}$.}

2018-02

Mantere , O , Saarela , M , Kieseppä , T, Raij , T , Mäntylä , T , Lindgren , M , Rikandi , E , Stoecker , W , Teegen , B \& Suvisaari , J 2018 , ' Anti-neuronal anti-bodies in patients with early psychosis ' , Schizophrenia Research , vol. 192 , pp. 404-407 . https://doi.org/10.1016/j.schres.2017.04.027

http://hdl.handle.net/10138/301089

https://doi.org/10.1016/j.schres.2017.04.027

publishedVersion

Downloaded from Helda, University of Helsinki institutional repository.

This is an electronic reprint of the original article.

This reprint may differ from the original in pagination and typographic detail.

Please cite the original version. 


\title{
Anti-neuronal anti-bodies in patients with early psychosis
}

\author{
O. Mantere ${ }^{\mathrm{a}, \mathrm{b}, *}$, M. Saarela $^{\mathrm{c}}$, T. Kieseppä ${ }^{\mathrm{d}}$, T. Raij $^{\mathrm{d}, \mathrm{e}}$, T. Mäntylä $^{\text {e,f,g }}$, M. Lindgren ${ }^{\mathrm{g}}$, \\ E. Rikandi e,g,h, W. Stoecker ${ }^{\text {i }}$, B. Teegen ${ }^{\mathrm{i}}$, J. Suvisaari ${ }^{\mathrm{g}}$ \\ a Department of Psychiatry, McGill University, Montréal, QC, Canada \\ b Bipolar Disorders Clinic, Douglas Mental Health University Institute, Montréal, QC, Canada \\ c Clinical Neurosciences, Neurology, University of Helsinki and Helsinki University Hospital, Helsinki, Finland \\ ${ }^{\mathrm{d}}$ Department of Psychiatry, Helsinki University and Helsinki University Hospital, Helsinki, Finland \\ e Department of Neuroscience and Biomedical Engineering, and Advanced Magnetic Imaging Center, Aalto NeuroImaging, Aalto University School of Science, Espoo, Finland \\ ${ }^{\mathrm{f}}$ VTT Technical Research Centre of Finland Ltd., Espoo, Finland \\ ${ }^{g}$ Mental Health Unit, National Institute for Health and Welfare, Helsinki, Finland \\ h Department of Psychology and Logopedics, Faculty of Medicine, University of Helsinki, Helsinki, Finland \\ ${ }^{i}$ Institute for Experimental Immunology, Euroimmun AG, Seekamp 31, 23560 Lübeck, Germany
}

\section{A R T I C L E I N F O}

\section{Article history:}

Received 21 December 2016

Received in revised form 11 April 2017

Accepted 14 April 2017

Available online 28 April 2017

\section{Keywords:}

$\mathrm{N}$-methyl D-aspartate (NMDA) receptor

Anti-glycine receptor

Anti-neuronal antibodies

Autoimmunity

Clinical high risk of psychosis

First episode psychosis

\begin{abstract}
A B S T R A C T
It may be challenging to distinguish autoimmune encephalitis associated with anti-neuronal autoantibodies from primary psychiatric disorders. Here, serum was drawn from patients with a first-episode psychosis $(n=70)$ or a clinical high-risk for psychosis $(n=6)$ and controls $(n=34)$. We investigated the serum prevalence of 24 antineuronal autoantibodies: IgG antibodies for anti- $\mathrm{N}$-methyl-D-aspartate-type glutamate receptor (anti-NMDAR), glutamate and $\gamma$-aminobutyric acid alpha and beta receptors (GABA-a, GABA-b), alpha-amino-3-hydroxy-5methyl-4-isoxazolepropionic acid receptor (AMPA), glycine receptor (GlyR), metabotropic glutamate receptor 1 and 5 (mGluR1, mGluR5), anti-Tr/Delta/notch-like epidermal growth factor-related receptor (DNER), contactin-associated protein-like 2 (CASPR2), myelin oligodendrocyte glycoprotein (MOG), glutamic acid decarboxylase-65 (GAD65), collapsin response mediator protein 5/crossveinless-2 (CV2), aquaporin-4 (AQP4), antidipeptidyl-peptidase-like protein-6 (DPPX), type 1 anti-neuronal nuclear antibody (ANNA-1, Hu), Ri, Yo, IgLON5, Ma2, zinc finger protein 4 (ZIC4), Rho GTPase-activating protein 26, amphiphysin, and recoverin, as well as IgA and IgM for dopamine-2-receptor (DRD2). Anti-NMDA IgG antibodies were positive with serum titer 1:320 in one patient with a clinical high risk for psychosis. He did not receive a diagnosis of encephalitis after comprehensive neurological evaluation. All other antineuronal autoantibodies were negative and there were no additional findings with immunohistochemistry of brain issues.
\end{abstract}

(c) 2017 Elsevier B.V. All rights reserved.

\section{Introduction}

Anti-N-methyl-D-aspartate-type glutamate receptor (anti-NMDAR) encephalitis was originally identified in young women with ovarian teratoma who had simultaneous prominent psychiatric manifestations. Later, it was noted that anti-NMDAR encephalitis can manifest without cancer (Dalmau et al., 2008), and some cases present with prominent psychotic symptoms (Kayser and Dalmau, 2016; Pollak et al., 2016). Clinically, patients with psychosis and IgG anti-NMDAR antibodies are

\footnotetext{
* Corresponding author at: Bipolar Disorders Clinic, Douglas Mental Health University Institute, Montréal, Canada, McGill University, Department of Psychiatry, Montréal, Canada, 6875 Boul Lasalle, Verdun, QC H4H 1R3, Canada.

E-mail addresses: outi.mantere@douglas.mcgill.ca (O. Mantere), mika.saarela@hus.fi (M. Saarela), Tuula.kieseppa@hus.fi (T. Kieseppä), tuukka.raij@hus.fi (T. Raij), teemu.mantyla@thl.fi (T. Mäntylä), Maija.lindgren@thl.fi (M. Lindgren), eva.rikandi@thl.fi (E. Rikandi),winfried.stoecker@euroimmun.de (W. Stoecker), b.teegen@euroimmun.de (B. Teegen), jaana.suvisaari@thl.fi (J. Suvisaari).
}

characterized by acute or subacute onset, catatonic symptoms, epilepsy and movement disorders including stereotypies, perseveration, and dystonia, or fever. Several other anti-neuronal antibodies targeting important ion channels, receptor-associated or regulatory molecules have been reported in some patients with psychosis and in patients with encephalitis presenting psychotic symptoms (Armangue et al., 2014; Heine et al., 2015; Leypoldt et al., 2015; Pollak et al., 2016). The prevalence of these antibodies in patients with an early psychosis remains open. The identification of encephalitis in patients with early psychosis is crucial, as over $75 \%$ of patients with classic anti-NMDAR encephalitis have substantial recovery with specific treatments, while antipsychotic treatment is not effective in these patients (Kayser and Dalmau, 2016).

In a sample of patients with a first-episode psychosis (FEP) or a clinical high-risk for psychosis ( $\mathrm{CHR}$ ) and controls, we investigated the prevalence of 24 anti-neuronal antibodies that have been associated with autoimmune encephalitis. 


\section{Methods}

\subsection{Clinical study protocol and assessment}

The study started in November 2010. FEP and CHR patients (age 18 to 40 years) were recruited from the catchment area of the Helsinki University Hospital, city of Hyvinkää, and City of Helsinki, Finland. The inclusion criteria for the FEP patients were a score of at least 4 in Unusual thought content or Hallucinations in the Brief Psychiatric Rating Scale - Extended (BPRS-E) (Ventura et al., 1993) and being fluent in Finnish. Patients with substance-induced psychotic disorders and psychotic disorders due to a general medical condition were excluded. Baseline assessment was conducted as soon as the patient had entered treatment and was able to give informed consent according to the treating personnel. Follow-ups included structured diagnostic interviews and a review of patient charts and were conducted at two and 12 months. CHR was assessed with the Structured Interview for Prodromal Syndromes (Miller et al., 2003), and the Criteria of Prodromal Syndromes (COPS) was used to define CHR status.

Controls, matched by age, sex and region of residence, were identified from the Population Register Center and assessed at baseline and 12 months with the same protocol as the patients. The study was carried out in accordance with the Code of Ethics of the World Medical Association (Declaration of Helsinki). The study protocol was approved by the Ethics Committee of the Helsinki University Hospital and by the institutional review boards of the National Institute for Health and Welfare, Helsinki, Finland. Research permissions were obtained from the Helsinki University Hospital and the Department of Psychiatry, and the Helsinki City Health Department. All participants gave a written informed consent.

\subsection{Laboratory analytical methods}

\subsubsection{Blood sampling}

A fasting blood sample was collected between 8 am and $10 \mathrm{am}$. Serum and plasma samples were immediately aliquoted and stored at $-80{ }^{\circ} \mathrm{C}$ with a maximal storage of 4 years. The frozen samples were all sent at the same time for analysis.

\subsubsection{Selection and testing of the antibodies}

We screened for anti-brain antibodies that had been described in either sporadic primary psychosis or in primary encephalitis with psychotic symptoms. We took into account anti-brain antibodies that were of potential interest based on genetic association studies in psychosis (Gatt et al., 2015; Nie et al., 2015). Based on a literature review (OM, JS, BT), we selected isotype IgG antibodies for NMDAR, glutamate and $\gamma$-aminobutyric acid alpha and beta receptors (GABA-a, GABA-b), alpha-amino-3-hydroxy-5-methyl-4-isoxazolepropionic acid receptor (AMPA), glycine receptor (GlyR), metabotropic glutamate receptor 1 and 5 (mGluR1, mGluR5), anti-Tr/Delta/notch-like epidermal growth factor-related receptor (DNER), contactin-associated protein-like 2 (CASPR2), myelin oligodendrocyte glycoprotein (MOG), glutamic acid decarboxylase-65 (GAD65), collapsin Response Mediator Protein 5/ Crossveinless-2 (CV2), aquaporin-4 (AQP4), anti-dipeptidyl-peptidaselike protein-6 (DPPX), type 1 anti-neuronal nuclear antibody (ANNA1, Hu), Ri, Yo, IgLON5, Ma2, zinc finger protein 4 (ZIC4), Rho GTPase-activating protein 26 , amphiphysin, and recoverin, as well as IgA and IgM antibodies for dopamine-2-receptor (DRD2).

Antibody analysis was performed blind to case/control status with an indirect immunofluorescence test of the immunoglobulin (Ig) IgG isotype, and additionally, IgA and IgM for DRD2. Sera were tested for the presence of antibodies using biochip mosaics of frozen brain sections (rat, monkey) and transfected HEK293 cells expressing the respective recombinant target antigens (Euroimmun, Germany) as previously described (Probst et al., 2014). Samples were classified as positive or negative based on fluorescence intensity of the transfected cells in direct comparison with non-transfected cells and control samples. Endpoint titers refer to the last dilution showing a detectable degree of fluorescence, with 1:10 being the cut-off for positivity. We report immunohistochemistry findings without corresponding positivity in the fixed cellbased assays, as these could have clinical relevance.

\subsection{Statistical analysis}

We calculated descriptive statistics for sociodemographic and clinical measures using SPSS Statistics for Windows, Version 22.0.

\section{Results}

The sociodemographic and clinical characteristics of the sample are described in Table 1 . The only anti-NMDAR positive patient (serum titer 1:320) was a CHR patient with a major depressive disorder. At one year, he had not converted to psychosis (See Table 2) but still had anti-NMDAR autoantibodies (serum titer 1:100) in his blood. After a thorough evaluation, clinical treatment of encephalitis was not indicated. All other cell-based assays were negative and there were also no additional findings with immunohistochemistry of brain tissues. Additionally, one patient with a brief psychotic episode was sent for neurological evaluation due to a clinical suspicion of encephalitis. In the clinical laboratory testing, he was found to have marginally elevated serum anti-glycine receptor antibodies (titer 1:10). Antibodies were not found in his cerebrospinal fluid and encephalitis and neurological diseases were excluded. At the follow-up of 12 months, the patient was in full remission (Table 2).

\section{Discussion}

This is the first study to report a person with a CHR for psychosis having an increased level of anti-NMDAR antibodies. All other mainly FEP patients showed a negative finding in the tested 24 anti-neuronal antibodies.

Our results further strengthen the previous view that if brain autoantibodies are systematically screened in patients primarily treated for a psychiatric diagnosis, sporadic cases screen antibody NMDAR positive (Kayser and Dalmau, 2016). Some samples including FEP patients have not detected NMDAR antibodies (Masdeu et al., 2012; Masopust et al., 2015), but others report individuals with positive findings (Lennox et al., 2017; Pathmanandavel et al., 2015; Steiner et al., 2013; Zandi et al., 2011). In the largest study so far (228 cases and 105 healthy controls) seven (3\%) patients but no controls had NMDAR antibodies (Lennox et al., 2017) However, the clinical relevance of the positive findings has to be confirmed by a thorough examination, and in this sample, none of the patients had a clinical diagnosis of an encephalitis. Other than anti-NMDAR antibodies, autoantibodies detected in autoimmune encephalitis seem to remain negative in patients with isolated early psychotic symptoms. Approximately $80 \%$ of adults with anti-NMDAR antibody encephalitis initially manifest behavioral and psychiatric symptoms (Kayser and Dalmau, 2016). The interest in anti-NMDAR antibodies has been strengthened by increased knowledge about the role of NMDAR in producing psychosis-like behavior and being involved in brain processes of etiological importance in psychosis (Carvajal et al., 2016; Pollak et al., 2016; Rosenthal-Simons et al., 2013). At the same time, isolated psychiatric symptoms, i.e. psychiatric symptoms without neurological symptoms, have been present in some patients with antiNMDAR encephalitis (Kayser et al., 2013). It is concluded that diagnostic evaluation in FEP can be focused to those presenting specific neurological symptoms (Kayser and Dalmau, 2016).

The strengths of the study include inclusion of FEP and high-risk patients, comprehensive selection of specific brain receptor antibodies, and a careful psychiatric evaluation. A limitation of the study was that no information about non-participants was available to evaluate the representativeness of the sample. Cerebrospinal fluid analysis, which 
Table 1

Characteristics of the sample with 76 cases and 34 controls.

\begin{tabular}{|c|c|c|c|}
\hline Characteristic & $\begin{array}{l}\text { Patients, } n= \\
70\end{array}$ & $\begin{array}{l}\text { Clinical high risk, } n= \\
6\end{array}$ & $\begin{array}{l}\text { Controls, } n= \\
34\end{array}$ \\
\hline Age, mean \pm standard deviation & $26.3 \pm 5.8$ & $25.4 \pm 3.5$ & $28.8 \pm 6.5$ \\
\hline Male, $n(\%)$ & $46(65.7 \%)$ & $5(83.3 \%)$ & $19(55.9 \%)$ \\
\hline \multicolumn{4}{|l|}{ Main lifetime diagnosis, $n(\%)$} \\
\hline Schizophrenia & $42(60.0 \%)$ & 0 & 0 \\
\hline Schizoaffective disorder & $2(2.9 \%)$ & 0 & 0 \\
\hline Bipolar disorder & $6(8.6 \%)$ & 0 & 0 \\
\hline Major depressive disorder & $2(2.9 \%)$ & $1(16.7 \%)$ & $6(17.6 \%)$ \\
\hline Psychosis NOS & $13(18.6 \%)$ & $1(16.7 \%)$ & 0 \\
\hline Other psychotic disorder & $5(7.1 \%)$ & 0 & 0 \\
\hline Other psychiatric diagnosis & 0 & $4(66.7 \%)$ & $5(14.7 \%)$ \\
\hline Brief Psychiatric Rating Scale score at baseline, mean \pm standard deviation & $44.5 \pm 10.4$ & $49.0 \pm 6.7$ & $25.5 \pm 2.9$ \\
\hline $\begin{array}{l}\text { Social and occupational functioning assessment scale score (Goldman et al., 1992) at baseline, mean } \pm \text { standard } \\
\text { deviation }\end{array}$ & $39.3 \pm 9.2$ & $50.3 \pm 7.1$ & $87.1 \pm 6.4$ \\
\hline Duration of antipsychotic treatment, median, days & 27 & 28 & NA \\
\hline
\end{tabular}

would confirm a pathologic process (Kreye et al., 2016), was not available for patients who did not have antibodies in peripheral blood, and therefore, false-negative findings are possible. The testing was performed using fixed cells; testing on living cells might be more sensitive (Gresa-Arribas et al., 2014) but is difficult to standardize and lacks suitability for large-scale analyses. The validation of these antibodies has been done only for neurological purposes. Future research

Table 2

Case reports for the two patients where NMDA encephalitis was suspected.

Case 1
Case 1 was a 23-year old university student with a history of recurrent major
depressive episodes. He was referred to the study from outpatient care as a
clinical high-risk patient. He had experiences of paranoid fears and illusions,
while the most difficult symptoms for the patient were depression, anxiety,
insomnia, and a rapid onset of difficulties in thinking and concentrating. He was
nonsmoking and had no history of alcohol or other substance abuse. He met the
criteria for Attenuated Positive Prodromal Syndrome by receiving a score of 4 in
suspiciousness and persecutory ideas. Baseline Social and occupational
functioning assessment scale score was 45 , and depressive symptoms were
severe (Beck Depression Inventory score 42 ). The sample was positive for
anti-NMDAR autoantibodies with serum titer $1: 320$. Based on this finding, we
contacted the treating personnel and the patient was sent for neurological
examination one year after the anti-NMDAR positive serum sample had been
taken. Serum anti-NMDAR autoantibodies were still present with titer $1: 100$, but
antibodies were not found in his cerebrospinal fluid and encephalitis and other
neurological diseases were excluded in the examination. Any etiology for the
presence of anti-NMDAR autoantibodies was not found. In the 1-year follow-up,
he no longer met clinical high-risk criteria but had a diagnosis of major
depressive disorder, with a severe major depressive episode without psychotic
features. His level of functioning was low and he had not been able to continue
his studies. He continued to have severe depressive and anxiety symptoms as
well as insomnia despite adequate pharmacological and psychological
treatment.

Case 2

Case 2 was a 34-year-old technician with no previous treatment contact for mental disorders. Psychotic symptoms, including delusions, disorganized behavior and marked variation between agitation and motor retardation developed within a few days. Low-grade fever and headache had preceded other symptoms a few days earlier. Treatment was started at a neurological department, where the patient was found to have marginally elevated serum anti-glycine receptor antibodies (titer 1:10). Antibodies were not found in his cerebrospinal fluid and thus, encephalitis and other neurological diseases were excluded. C-reactive protein, lipid, glucose and liver function tests were normal. Laboratory tests for drug use were negative, and the patient had no history of smoking or current alcohol use. Intensity of delusions at the most severe phase of the illness was 7 (extremely severe) and intensity of hallucinations 5 (moderately severe) based on baseline Brief Psychiatric Rating Scale interview. Treatment was continued at a psychiatric department, and psychotic symptoms resolved within a month. At the two-month follow-up, the following diagnoses were assigned: a brief psychotic disorder, with simultaneous social phobia (current) and previous alcohol abuse, in full remission. At one year, the patient had returned to work in full remission of psychotic symptoms. should explore the possibility that there are other anti-neuronal autoantibodies that are specifically related to psychotic disorders.

\section{Conflict of interest}

All authors declare that they have no conflicts of interest.

\section{Contributors}

OM, JS, TR, TK, TM, ML, and ER contributed to design and sampling of the FEP and CHR samples and controls. OM, JS and MS designed the study and wrote the protocol concerning autoantibodies. BT and WS were responsible for the analysis of the antibodies. $\mathrm{OM}$ and JS managed the literature searches. OM undertook the statistical analysis, and wrote the first draft of the manuscript. All authors contributed to and have approved the final manuscript.

\section{Acknowledgements}

This study was funded by the Helsinki University Central Hospital (Outi Mantere) (Funding Reference Numbers: TYH2013332; TYH2014228), Academy of Finland Grants 129434 and 278171, and a Sigrid Juselius Foundation grant (Jaana Suvisaari).

\section{References}

Armangue, T., Leypoldt, F., Dalmau, J., 2014. Autoimmune encephalitis as differential diagnosis of infectious encephalitis. Curr. Opin. Neurol. 27 (3), 361-368.

Carvajal, F.J., Mattison, H.A., Cerpa, W., 2016. Role of NMDA receptor-mediated Glutamatergic signaling in chronic and acute Neuropathologies. Neural Plast 2016 2701526.

Dalmau, J., Gleichman, A.J., Hughes, E.G., Rossi, J.E., Peng, X., Lai, M., Dessain, S.K. Rosenfeld, M.R. Balice-Gordon, R. Lynch, D.R. 2008. Anti-NMDA-receptor encephalitis: case series and analysis of the effects of antibodies. Lancet Neurol. 7 (12), 1091-1098.

Gatt, J.M., Burton, K.L., Williams, L.M., Schofield, P.R., 2015. Specific and common genes implicated across major mental disorders: a review of meta-analysis studies. J. Psychiatr. Res. 60, 1-13.

Goldman, H.H., Skodol, A.E., Lave, T.R., 1992. Revising axis V for DSM-IV: A review of measures of social functioning. Am. J. Psychiatry 149, 1148-1156.

Gresa-Arribas, N., Titulaer, M.J., Torrents, A., Aguilar, E., McCracken, L., Leypoldt, F. Gleichman, A.J., Balice-Gordon, R., Rosenfeld, M.R., Lynch, D., Graus, F., Dalmau, J., 2014. Antibody titres at diagnosis and during follow-up of anti-NMDA receptor encephalitis: a retrospective study. Lancet Neurol. 13 (2), 167-177.

Heine, J., Pruss, H., Bartsch, T., Ploner, C.J., Paul, F., Finke, C., 2015. Imaging of autoimmune encephalitis-relevance for clinical practice and hippocampal function. Neuroscience 309, 68-83.

Kayser, M.S., Dalmau, J., 2016. Anti-NMDA receptor encephalitis, autoimmunity, and psychosis. Schizophr. Res. 176 (1), 36-40.

Kayser, M.S., Titulaer, M.J., Gresa-Arribas, N., Dalmau, J., 2013. Frequency and characteristics of isolated psychiatric episodes in anti-N-methyl-d-aspartate receptor encephalitis. JAMA Neurol. 70 (9), 1133-1139.

Kreye, J., Wenke, N.K., Chayka, M., Leubner, J., Murugan, R., Maier, N., Jurek, B., Ly, L.T. Brandl, D., Rost, B.R., Stumpf, A., Schulz, P., Radbruch, H., Hauser, A.E., Pache, F., Meisel, A., Harms, L., Paul, F., Dirnagl, U., Garner, C., Schmitz, D., Wardemann, H., Pruss, H., 2016. Human cerebrospinal fluid monoclonal N-methyl-D-aspartate receptor autoantibodies are sufficient for encephalitis pathogenesis. Brain 139 (Pt 10), 2641-2652.

Lennox, B.R., Palmer-Cooper, E.C., Pollak, T., Hainsworth, J., Marks, J., Jacobson, L., Lang, B., Fox, H., Ferry, B., Scoriels, L., Crowley, H., Jones, P.B., Harrison, P.J., Vincent, A., team, P.P.s., 2017. Prevalence and clinical characteristics of serum neuronal cell surface 
antibodies in first-episode psychosis: a case-control study. Lancet Psychiatry 4 (1), 42-48.

Leypoldt, F., Armangue, T., Dalmau, J., 2015. Autoimmune encephalopathies. Ann. N. Y. Acad. Sci. 1338, 94-114.

Masdeu, J.C., Gonzalez-Pinto, A., Matute, C., Ruiz De Azua, S., Palomino, A., De Leon, J., Berman, K.F., Dalmau, J., 2012. Serum IgG antibodies against the NR1 subunit of the NMDA receptor not detected in schizophrenia. Am. J. Psychiatry 169 (10), 1120-1121.

Masopust, J., Andrys, C., Bazant, J., Vysata, O., Kuca, K., Valis, M., 2015. Anti-NMDA receptor antibodies in patients with a first episode of schizophrenia. Neuropsychiatr. Dis. Treat. 11, 619-623.

Miller, T.J., McGlashan, T.H., Rosen, J.L., Cadenhead, K., Cannon, T., Ventura, J., McFarlane, W., Perkins, D.O., Pearlson, G.D., Woods, S.W., 2003. Prodromal assessment with the structured interview for prodromal syndromes and the scale of prodromal symptoms: predictive validity, interrater reliability, and training to reliability. Schizophr. Bull. 29 (4), 703-715.

Nie, F., Wang, X., Zhao, P., Yang, H., Zhu, W., Zhao, Y., Chen, B., Valenzuela, R.K., Zhang, R. Gallitano, A.L., Ma, J., 2015. Genetic analysis of SNPs in CACNA1C and ANK3 gene with schizophrenia: a comprehensive meta-analysis. Am. J. Med. Genet. B Neuropsychiatr. Genet. 168 (8), 637-648.

Pathmanandavel, K., Starling, J., Merheb, V., Ramanathan, S., Sinmaz, N., Dale, R.C., Brilot, F., 2015. Antibodies to surface dopamine-2 receptor and N-methyl-D-aspartate receptor in the first episode of acute psychosis in children. Biol. Psychiatry 77 (6), 537-547.
Pollak, T.A., Beck, K., Irani, S.R., Howes, O.D., David, A.S., McGuire, P.K., 2016. Autoantibodies to central nervous system neuronal surface antigens: psychiatric symptoms and psychopharmacological implications. Psychopharmacology 233 (9), 1605-1621.

Probst, C., Saschenbrecker, S., Stoecker, W., Komorowski, L., 2014. Anti-neuronal autoantibodies: current diagnostic challenges. Mult. Scler. Relat. Disord. 3 (3), 303-320.

Rosenthal-Simons, A., Durrant, A.R., Heresco-Levy, U., 2013. Autoimmune-induced glutamatergic receptor dysfunctions: conceptual and psychiatric practice implications. Eur. Neuropsychopharmacol. 23 (12), 1659-1671.

Steiner, J., Walter, M., Glanz, W., Sarnyai, Z., Bernstein, H.G., Vielhaber, S., Kastner, A., Skalej, M., Jordan, W., Schiltz, K., Klingbeil, C., Wandinger, K.P., Bogerts, B., Stoecker, W., 2013. Increased prevalence of diverse $\mathrm{N}$-methyl-D-aspartate glutamate receptor antibodies in patients with an initial diagnosis of schizophrenia: specific relevance of IgG NR1a antibodies for distinction from N-methyl-D-aspartate glutamate receptor encephalitis. JAMA Psychiatry 70 (3), 271-278.

Ventura, J. Lukoff, D., Nuechterlein, K.H. Liberman, R.P., Green, M.F., Shaner, A, 1993. Brief Psychiatric Rating Scale (BPRS), expanded version (4.0): scales, anchor points, and.administration manual. Int. J. Methods Psychiatr. Res. 3, 227-243.

Zandi, M.S., Irani, S.R., Lang, B., Waters, P., Jones, P.B., McKenna, P., Coles, A.J., Vincent, A., Lennox, B.R., 2011. Disease-relevant autoantibodies in first episode schizophrenia. J. Neurol. 258 (4), 686-688. 